\title{
Electron acoustic solitons in the presence of an electron beam and superthermal electrons
}

\author{
S. Devanandhan ${ }^{1}$, S. V. Singh ${ }^{1,2}$, G. S. Lakhina ${ }^{1}$, and R. Bharuthram ${ }^{3}$ \\ ${ }^{1}$ Indian Institute of Geomagnetism, New Panvel (West), Navi Mumbai, India \\ ${ }^{2}$ School of Physics, University of Kwazulu-Natal, Durban, South Africa \\ ${ }^{3}$ University of the Western Cape, Bellville, South Africa
}

Received: 22 May 2011 - Revised: 5 September 2011 - Accepted: 12 September 2011 - Published: 23 September 2011

\begin{abstract}
Arbitrary amplitude electron acoustic solitons are studied in an unmagnetized plasma having cold electrons and ions, superthermal hot electrons and an electron beam. Using the Sagdeev pseudo potential method, theoretical analysis is carried out by assuming superthermal hot electrons having kappa distribution. The results show that inclusion of an electron beam alters the minimum value of spectral index, $\kappa$, of the superthermal electron distribution and Mach number for which electron-acoustic solitons can exist and also changes their width and electric field amplitude. For the auroral region parameters, the maximum electric field amplitudes and soliton widths are found in the range $\sim(30-524) \mathrm{mV} \mathrm{m}^{-1}$ and $\sim(329-729) \mathrm{m}$, respectively, for fixed Mach number $M=1.1$ and for electron beam speed of (660-1990) $\mathrm{km} \mathrm{s}^{-1}$.
\end{abstract}

\section{Introduction}

Electron acoustic mode can exist in a plasma having two electron components, hot and cold, and it propagates at a speed close to the electron-acoustic speed. This mode is very useful in understanding the high frequency component of broadband electrostatic noise observed in different regions of the earth's magnetosphere. Electron-acoustic solitary waves have been observed in the Earth' magnetosphere by various satellites, e.g. Viking, FAST etc. (Dubouloz et al., 1991, 1993; Cattell et al., 1998; Pottelette et al., 1999; Ergun et al., 1999; Miyake et al., 2000).

Several theoretical studies have been done on electronacoustic solitary waves in multi-component space plasmas (Mace et al., 1991; Mace and Hellberg, 2001; Berthomier et al., 2000, 2003). Singh et al. (2001) examined electron acoustic solitary waves in a four-component plasma and applied their results to explain the Viking satellite observations

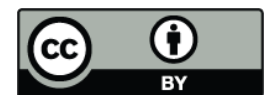

Correspondence to: S. V. Singh

(satyavir@iigs.iigm.res.in) in the dayside auroral zone. Verheest et al. $(2005,2007)$ showed that inclusion of hot electron inertia can lead to positive potential electron-acoustic solitons. Lakhina et al. (2008a, b) investigated large amplitude ion- and electronacoustic solitary waves in an unmagnetized multi-fluid plasmas. They found that in four-component plasma consisting of cold background electrons and ions, a hot electron beam and a hot ion beam, three types of solitary waves, namely, slow ion-acoustic, ion-acoustic and electron-acoustic solitons are possible. Co-existence of positive and negative potential solitary structures were studied by Kakad et al. (2007, 2009) in multispecies, unmagnetized plasmas by using reductive perturbation and pseudo-potential methods. Lakhina et al. $(2009,2011)$ proposed a model based on electronacoustic solitons and double layers in a four-component plasma system consisting of core electrons, two counter streaming electron beams, and one type of ions, to explain the electrostatic solitary waves observed by CLUSTER satellite in the magnetosheath region (Pickett et al., 2005). Several other studies have been done on electron-acoustic solitons using non-thermal distribution for the hot electron component (Singh and Lakhina, 2004; Gill et al., 2006; El-Shewy, 2007).

All the theoretical studies discussed above considered hot electron-component in their model either as a Maxwellian, or included the electron inertia effects. However, it is often observed that in space plasmas the distribution functions deviate from the Maxwellian due to the presence of superthermal particles having high energy tails (Vasyliunas, 1968; Leubner, 1982; Marsch et al., 1982; Armstrong et al., 1983). These superthermal particles can be described by $\kappa$ distribution rather than Maxwellian or any other nonthermal distribution (Summers and Thorne, 1991; Thorne and Summers, 1991a, b; Thorne and Horne, 1994; Mace and Hellberg, 1995, Hellberg and Mace, 2002).

Only a few studies have been done on electron-acoustic solitary waves using $\kappa$-distribution for hot electrons. For example, Younsi et al. (2010) studied the existence of arbitrary

Published by Copernicus Publications on behalf of the European Geosciences Union and the American Geophysical Union. 
amplitude electron-acoustic solitary potentials in a plasma having stationary ions, cold inertial electrons and hot superthermal electrons. They found that as spectral index $\kappa$ decreases, soliton amplitude increases while its width narrows down. Sahu (2010) studied the existence of small amplitude electron-acoustic double layers in an unmagnetized plasma in a theoretical model similar to Younsi et al. (2010). Both the above studies did not include the effect of cold electron temperature and mobile ions. Devanandhan et al. (2011) extended their work to include the thermal effects of the cool electrons and mobile ions on electron-acoustic solitary waves. It is found that inclusion of finite temperature of the cold electrons alters the existence regime of the solitons and there is a significant decrease in solitary electric field amplitude with an increase in the temperature of cold electrons.

Satellite observations in different regions of the Earth's magnetosphere have shown that the electrostatic solitary waves are generally associated with electron or/and ion beams (Dubouloz et al., 1991, 1993; Cattell et al., 1998). So far in the literature, the studies on the electron-acoustic solitary waves involving $\kappa$-distribution have not taken into account the effect of electron/ion beams. The aim of this paper is to study the effects of electron beam on electronacoustic solitary waves. We extend the theoretical model of Devanandhan et al. (2011) to four component plasma by adding an electron beam and study its effects on the evolution of electron-acoustic solitary waves. The organization of the paper is as follows. In Sect. 2 we describe the theoretical plasma model and derive the Sagdeev pseudo potential for arbitrary amplitude electron acoustic waves in four-component unmagnetized plasma consisting of cold electrons, superthermal electrons, electron beam and ions. In Sect. 3 we present numerical results of the theoretical model. Results are summarized in the last section.

\section{Theoretical model}

An infinite, homogeneous, collisionless, unmagnetized, fourcomponent plasma is considered having fluid cold electrons, kappa distributed hot electrons, an electron beam and fluid ions. We consider the waves propagating along $\mathrm{x}$-direction. The normalized multi-fluid equations of continuity, momentum, and equation of state which governs the dynamics of cold electrons, beam electrons and ions are given by,

$$
\begin{aligned}
& \frac{\partial n_{j}}{\partial t}+\frac{\partial}{\partial x}\left(n_{j} v_{j}\right)=0 \\
& \frac{\partial v_{j}}{\partial t}+v_{j} \frac{\partial v_{j}}{\partial x}+\frac{1}{\mu_{j} n_{j}} \frac{\partial P_{j}}{\partial x}-\frac{Z_{j}}{\mu_{j}} \frac{\partial \phi}{\partial x}=0 \\
& \frac{\partial P_{j}}{\partial t}+v_{j} \frac{\partial P_{j}}{\partial x}+3 P_{j} \frac{\partial v_{j}}{\partial x}=0
\end{aligned}
$$

where the subscript $j=\mathrm{c}, \mathrm{b}$, i represents cold electrons, beam electrons and ions, respectively, $Z_{j}= \pm 1$ for electrons and ions respectively, and $\mu_{j}=m_{j} / m_{\mathrm{e}}$. Here $m_{j}, v_{j}, P_{j}, n_{j}$ and $\phi_{j}$ denote the mass, velocity, thermal pressure, number density and electrostatic potential for $j$-th species. Furthermore, we have assumed the same adiabatic index, i.e. $\gamma=3$, for all the species in the equation of state given by Eq. (3). This is justified as we are considering one dimensional problem in an unmagnetized plasma, and the particles will essentially have one degree of freedom along the direction of wave propagation.

In Eqs. (1)-(3), the densities, velocities, lengths, temperature, time, electrostatic potential $(\varphi)$, and thermal pressure are normalized by total electron density $N_{\mathrm{o}}=N_{\mathrm{oh}}+N_{\mathrm{oc}}+$ $N_{\mathrm{ob}}=N_{\mathrm{oi}}$, thermal velocity of hot electrons $v_{\text {th }}=\sqrt{T_{\mathrm{h}} / m_{\mathrm{e}}}$, effective hot electron Debye length $\lambda_{\mathrm{dh}}=\sqrt{T_{\mathrm{h}} / 4 \pi N_{\mathrm{o}} e^{2}}$, hot electron temperature $T_{\mathrm{h}}$, inverse of electron plasma frequency $\omega_{\mathrm{pe}}^{-1}=\sqrt{m_{\mathrm{e}} / 4 \pi N_{\mathrm{o}} e^{2}}, T_{\mathrm{h}} / e$ and $N_{\mathrm{o}} T_{\mathrm{h}}$, respectively.

The Eqs. (1)-(3) are coupled through the Poisson equation which is given as,

$$
\frac{\partial^{2} \phi}{\partial x^{2}}=n_{\mathrm{h}}+n_{\mathrm{c}}+n_{\mathrm{b}}-n_{\mathrm{i}}
$$

Here $n_{\mathrm{h}}$ represents the number density of hot electrons. The standard three dimensional isotropic kappa distribution function of hot electrons is given by Thorne and Summers (1991a),

$f_{\text {oh }}(v)=\frac{N_{\text {oh }}}{\pi^{\frac{3}{2}} \theta^{3}} \frac{\Gamma(\kappa)}{\sqrt{\kappa} \Gamma(\kappa-1 / 2)}\left(1+\frac{v^{2}}{\kappa \theta^{2}}\right)^{-(\kappa+1)}$

where $\Gamma(\kappa)$ is the gamma function and $\theta$ is a modified electron thermal speed given by $\theta=\sqrt{(2-3 / \kappa)\left(T_{\mathrm{h}} / m_{\mathrm{e}}\right),} \kappa$ is the spectral index, $N_{\mathrm{oh}}, T_{\mathrm{h}}, m_{\mathrm{e}}$ and $v$ are the equilibrium density, equilibrium temperature, mass and velocity of the hot electrons, respectively. In the limit $\kappa \rightarrow \infty$ modified thermal speed $\theta$ reduces to $\sqrt{ } 2 v_{\text {th }}$, where $v_{\text {th }}=\sqrt{T_{\mathrm{h}} / m_{\mathrm{e}}}$ is the usual electron thermal speed. In order to have physically meaningful thermal speed one requires $\kappa>3 / 2$. The kappa distribution function reduces to Maxwellian distribution when $\kappa \rightarrow \infty$. Replacing $\frac{v^{2}}{\theta^{2}}$ by $\frac{v^{2}}{\theta^{2}}-\frac{2 \varphi e}{m \theta^{2}}$ in the distribution function and integrating over velocity space the normalized number density for hot electrons can be obtained as

$n_{\mathrm{h}}=n_{\mathrm{oh}}\left(1-\frac{\phi}{(\kappa-3 / 2)}\right)^{-(\kappa-1 / 2)}$

The linear dispersion relation can be obtained by linearizing and solving the set of Eqs. (1)-(6) and is given by,

$$
\begin{aligned}
& 1+\frac{1}{k^{2} \lambda_{\mathrm{dh}}^{2}}\left(\frac{\kappa-1 / 2}{\kappa-3 / 2}\right)-\frac{\omega_{\mathrm{pc}}^{2}}{\left(\omega^{2}-3 k^{2} v_{\mathrm{tc}}^{2}\right)} \\
& -\frac{\omega_{\mathrm{pb}}^{2}}{\left(\left(\omega-k v_{\mathrm{o}}\right)^{2}-3 k^{2} v_{\mathrm{tb}}^{2}\right)}-\frac{\omega_{\mathrm{pi}}^{2}}{\left(\omega^{2}-3 k^{2} v_{\mathrm{ti}}^{2}\right)}=0
\end{aligned}
$$

where $\omega_{\mathrm{pj}}=\sqrt{4 \pi N_{\mathrm{o} j} e^{2} / m_{j}}$ and $v_{\mathrm{t} j}=\left(T_{j} / m_{j}\right)^{1 / 2}$ are the plasma frequency and thermal speed of the $j$-th species, respectively. Here $T_{j}$ and $m_{j}$ are the temperature and mass of 
the $j$-th species. Further, assuming electron beam drift speed $v_{\mathrm{o}}=0, N_{\mathrm{oh}} T_{\mathrm{b}} / N_{\mathrm{oc}} T_{\mathrm{h}} \ll 1$ (low beam temperature compared to hot electron temperature) and $N_{\mathrm{ob}} T_{\mathrm{c}} / N_{\mathrm{oc}} T_{\mathrm{b}} \ll 1$ (low beam density compared to cold electron density), and neglecting ion effects, Eq. (7) can be simplified to give the following dispersion relation for the electron-acoustic waves,

$$
\omega^{2}=\frac{k^{2} v_{\mathrm{th}}^{2}\left(\frac{N_{\mathrm{oc}}}{N_{\mathrm{oh}}}\right)\left(\frac{\kappa-3 / 2}{\kappa-1 / 2}\right)}{1+\lambda_{\mathrm{dh}}^{2} k^{2}\left(\frac{\kappa-3 / 2}{\kappa-1 / 2}\right)}\left\{1+3 k^{2} \lambda_{\mathrm{dc}}^{2}+\frac{N_{\mathrm{ob}}}{N_{\mathrm{oc}}}\left[1+3 k^{2} \lambda_{\mathrm{db}}^{2}\right]\right\}
$$

In the absence of electron beam, i.e. $N_{\mathrm{ob}}=0$, the dispersion relation (8) reduces to that of Mace et al. (1999). For $\kappa \rightarrow \infty$, the dispersion relation (8) is the same as obtained by Singh et al. (2001). Further, it reduces to the dispersion relation obtained by Berthomier et al. (2000) for $\kappa \rightarrow \infty$ and $v_{\mathrm{o}}=0$.

Equations (1)-(4) are transformed to a set of equations that depend on a single variable $\xi$ by using the transformation $\xi=x-M \mathrm{t}$, where $M=V / v_{\text {th }}$ is the Mach number defined with respect to the usual hot electron thermal speed $v_{\text {th }}$, and $V$ is the velocity of the solitons. This normalization is generally used in the literature (Mace and Hellberg, 2001). The transformed equations can be written as

$$
\begin{aligned}
& -M \frac{\partial n_{j}}{\partial \xi}+\frac{\partial}{\partial \xi}\left(n_{j} v_{j}\right)=0 \\
& -M \frac{\partial v_{j}}{\partial \xi}+v_{j} \frac{\partial v_{j}}{\partial \xi}+\frac{1}{\mu_{j} n_{j}} \frac{\partial P_{j}}{\partial \xi}-\frac{Z_{j}}{\mu_{j}} \frac{\partial \phi}{\partial \xi}=0 \\
& -M \frac{\partial P_{j}}{\partial \xi}+v_{j} \frac{\partial P_{j}}{\partial \xi}+3 P_{j} \frac{\partial v_{j}}{\partial \xi}=0 \\
& \frac{\partial^{2} \phi}{\partial \xi^{2}}=n_{\mathrm{h}}+n_{\mathrm{c}}+n_{\mathrm{b}}-n_{\mathrm{i}}
\end{aligned}
$$

Using appropriate boundary conditions along with $\phi=0$ and $d \phi / d \xi=0$ at $\xi \rightarrow \pm \infty$ Eqs. (9)-(12) can be integrated to yield the energy integral,

$$
\frac{1}{2}\left(\frac{d \phi}{d \xi}\right)^{2}+V(\phi, M)=0
$$

where

$$
\begin{aligned}
& V(\phi, M)=n_{\mathrm{oh}}\left[1-\left(1-\frac{\phi}{\kappa-3 / 2}\right)^{-(\kappa-3 / 2)}\right] \\
& +n_{\mathrm{oc}}\left[M^{2}-\frac{M}{\sqrt{2}}\left\{M^{2}+3 \sigma_{\mathrm{c}}+2 \phi+\sqrt{\left(M^{2}+3 \sigma_{\mathrm{c}}+2 \phi\right)^{2}-12 \sigma_{\mathrm{c}} M^{2}}\right\}^{1 / 2}\right] \\
& +n_{\mathrm{oc}} \sigma_{\mathrm{c}}\left[1-2 \sqrt{2} M^{3}\left\{M^{2}+3 \sigma_{\mathrm{c}}+2 \phi+\sqrt{\left(M^{2}+3 \sigma_{\mathrm{c}}+2 \phi\right)^{2}-12 \sigma_{\mathrm{c}} M^{2}}\right\}^{-3 / 2}\right] \\
& +n_{\mathrm{ob}}\left[\left(M-v_{\mathrm{o}}\right)^{2}-\frac{\left(M-v_{\mathrm{o}}\right)}{\sqrt{2}}\left\{\left(M-v_{\mathrm{o}}\right)^{2}+3 \sigma_{\mathrm{b}}+2 \phi\right.\right. \\
& \left.\left.+\sqrt{\left(\left(M-v_{\mathrm{o}}\right)^{2}+3 \sigma_{\mathrm{b}}+2 \phi\right)^{2}-12 \sigma_{\mathrm{b}}\left(M-v_{\mathrm{o}}\right)^{2}}\right\}^{1 / 2}\right] \\
& +n_{\mathrm{ob}} \sigma_{\mathrm{b}}\left[1-2 \sqrt{2}\left(M-v_{\mathrm{o}}\right)^{3}\left\{\left(M-v_{\mathrm{o}}\right)^{2}+3 \sigma_{\mathrm{b}}+2 \phi\right.\right.
\end{aligned}
$$

$$
\begin{aligned}
& \left.\left.+\sqrt{\left(\left(M-v_{\mathrm{o}}\right)^{2}+3 \sigma_{\mathrm{b}}+2 \phi\right)^{2}-12 \sigma_{\mathrm{b}}\left(M-v_{\mathrm{o}}\right)^{2}}\right\}^{-3 / 2}\right] \\
& +\mu_{\mathrm{i}}\left[M^{2}-\frac{M}{\sqrt{2}}\left\{M^{2}+\frac{3 \sigma_{\mathrm{i}}}{\mu_{\mathrm{i}}}-\frac{2 \phi}{\mu_{\mathrm{i}}}+\sqrt{\left(M^{2}+\frac{3 \sigma_{\mathrm{i}}}{\mu_{\mathrm{i}}}-\frac{2 \phi}{\mu_{\mathrm{i}}}\right)^{2}-\frac{12 \sigma_{\mathrm{i}} M^{2}}{\mu_{\mathrm{i}}}}\right\}^{1 / 2}\right] \\
& +\sigma_{\mathrm{i}}\left[1-2 \sqrt{2} M^{3}\left\{M^{2}+\frac{3 \sigma_{\mathrm{i}}}{\mu_{\mathrm{i}}}-\frac{2 \phi}{\mu_{\mathrm{i}}}+\sqrt{\left(M^{2}+\frac{3 \sigma_{\mathrm{i}}}{\mu_{\mathrm{i}}}-\frac{2 \phi}{\mu_{\mathrm{i}}}\right)^{2}-\frac{12 \sigma_{\mathrm{i}} M^{2}}{\mu_{\mathrm{i}}}}\right\}^{-3 / 2}\right]
\end{aligned}
$$

where $\sigma_{j}=T_{j} / T_{\mathrm{h}}$ is the ratio of the temperature of $j$-th species to hot electron temperature. Solitary wave solutions can be obtained from Eq. (13) when the Sagdeev potential $V(\phi, M)$ satisfies the following conditions: $V(\phi, M)=$ $0, d V(\phi, M) / d \phi=0$, and $d^{2} V(\phi, M) / d \phi^{2}<0$ at $\phi=$ $0 ; V(\phi, M)=0$ at $\phi=\phi_{\mathrm{m}}$ and $V(\phi, M)<0$ for $0<|\phi|<$ $\left|\phi_{\mathrm{m}}\right|, \phi_{\mathrm{m}}$ is the maximum potential. From Eq. (14) it is seen that the conditions $V(\phi, M)=d V(\phi, M) / d \phi=$ 0 at $\phi=0$ are automatically satisfied. The condition $d^{2} V(\phi, M) / d \phi^{2}<0$ at $\phi=0$ is satisfied provided $M>M_{\mathrm{o}}$, where $M_{\mathrm{o}}$ is the critical Mach number and satisfies the following equation

$$
\begin{aligned}
& \frac{n_{\mathrm{oc}}}{\left(M_{0}^{2}-3 \sigma_{\mathrm{c}}\right)}+\frac{n_{\mathrm{ob}}}{\left(\left(M_{\mathrm{o}}-v_{\mathrm{o}}\right)^{2}-3 \sigma_{\mathrm{b}}\right)} \\
& +\frac{1}{\mu_{\mathrm{i}}\left(M_{\mathrm{o}}^{2}-\frac{3 \sigma_{\mathrm{i}}}{\mu_{\mathrm{i}}}\right)}=n_{\mathrm{oh}}\left(\frac{\kappa-1 / 2}{\kappa-3 / 2}\right)
\end{aligned}
$$

Equation (15) yields 6 roots but all the roots will not be physical. For numerical computations only the real positive roots for the critical Mach numbers will be considered. For the chosen parameters for numerical computation, Eq. (15) yields three negative and three positive roots. Two of the positive roots are very small (of the order of $10^{-3}$ and $10^{-1}$ ) and one root is of the order of unity. We are interested in the positive root which is closer to unity and describes the electron-acoustic mode. The soliton solutions are obtained for subsonic ( $\left.V<\sqrt{ } 2 v_{\text {th }}\right)$ hot electrons; where $\sqrt{ } 2 v_{\text {th }}$ is the effective thermal velocity of the hot electrons in the limit $\kappa \rightarrow \infty$. All other species are supersonic, i.e. $V>v_{\mathrm{tc}}, v_{\mathrm{tb}}, v_{\mathrm{ti}}$.

\section{Numerical results}

For numerical computations, we use the auroral region parameters from Dubouloz et al. $(1991,1993)$ paper. These parameters corresponds to an observed event and are as follows: cold electron density $N_{\mathrm{oc}}=0.2 \mathrm{~cm}^{-3}$, hot electron density $N_{\mathrm{oh}}=1.5 \mathrm{~cm}^{-3}$, beam electron density $N_{\mathrm{ob}}=1.0 \mathrm{~cm}^{-3}$ and normalized beam velocity $v_{\mathrm{o}} / v_{\text {th }}=0.1$, cold to hot electron temperature ratio, ion to hot electron temperature ratio are $\sigma_{\mathrm{c}}=T_{\mathrm{c}} / T_{\mathrm{h}}=T_{\mathrm{i}} / T_{\mathrm{h}}\left(=\sigma_{\mathrm{i}}\right)=0.001$ and beam to hot electron temperature ratio, $\sigma_{\mathrm{b}}=T_{\mathrm{b}} / T_{\mathrm{h}}=0.01$. The value of the spectral index $\kappa$ for the hot electron is not given by Dubouloz et al. (1991, 1993). We take typically $\kappa=4$ for the hot electron component. For the chosen parameters mentioned above, the inequalities used in Eq. (7) to obtain dispersion relation (8) 


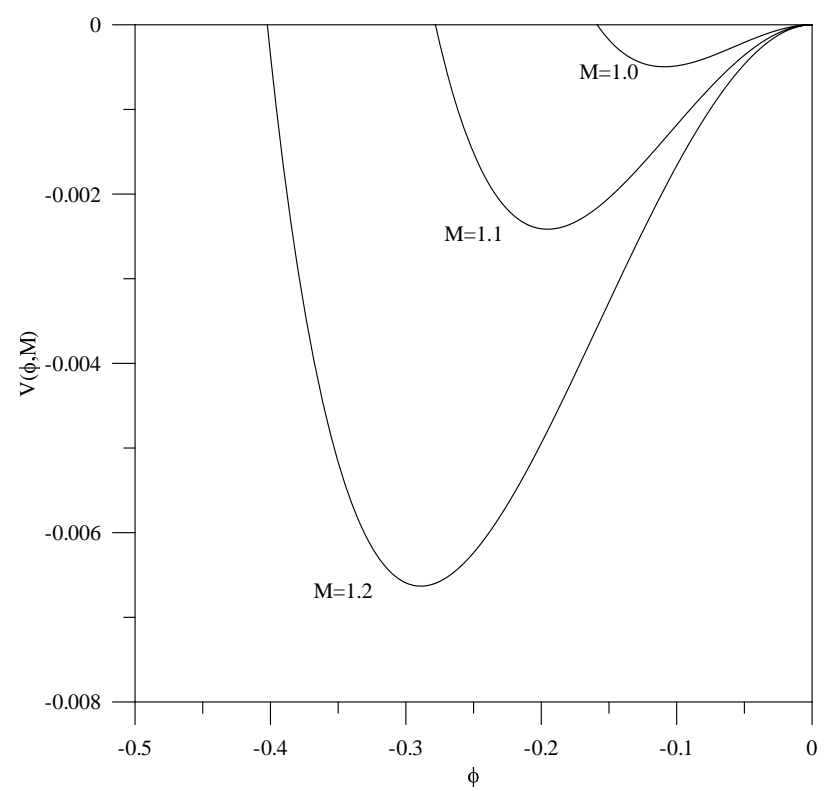

Fig. 1. Variation of Sagdeev potential $V(\phi, M)$ vs potential $\phi$ for the parameters, $\kappa=4, N_{\mathrm{oc}}=0.2 \mathrm{~cm}^{-3}, N_{\mathrm{oh}}=1.5 \mathrm{~cm}^{-3}, N_{\mathrm{ob}}=$ $1.0 \mathrm{~cm}^{-3}, v_{\mathrm{o}} / v_{\mathrm{th}}=0.1, \sigma_{\mathrm{c}}=T_{\mathrm{c}} / T_{\mathrm{h}}=T_{\mathrm{i}} / T_{\mathrm{h}}\left(=\sigma_{\mathrm{i}}\right)=0.001$ and $\sigma_{\mathrm{b}}=T_{\mathrm{b}} / T_{\mathrm{h}}=0.01$ for Mach numbers $M=1.0,1.1$ and 1.2 respectively.

for electron-acoustic waves have the values $N_{\mathrm{oh}} T_{\mathrm{b}} / N_{\mathrm{oc}} T_{\mathrm{h}}=$ 0.075 and $N_{\mathrm{ob}} T_{\mathrm{c}} / N_{\mathrm{oc}} T_{\mathrm{b}}=0.5$. Since these values are below 1 , we are confident that the mode we are studying is the electron-acoustic mode, possibly slightly modified by the presence of the electron beam. Equations (13) and (14) are solved numerically to obtain electrostatic potential $(\phi)$ profiles and Sagdeev potential $V(\phi, M)$, respectively.

Figure 1 shows the variation of Sagdeev potential $V(\phi, M)$ with normalized potential $\phi$ for various values of $M$ for the parameters mentioned above. The points where the curves cut the $\mathrm{x}$-axis define the value of the potential $\phi$ which is the solution of $V(\phi, M)=0$. Soliton solution exists for the Mach number ranging from $0.88<M<1.28$. It is evident from the figure that soliton amplitudes increase with the increase in Mach number. Corresponding normalized electric field $\left(E_{\mathrm{N}}\right)$ profiles, which are bipolar in nature, have been plotted in Fig. 2.

Next, the effect of beam parameters such as electron beam temperature and speed are studied on the evolution of electrostatic solitary potential structures. Figure 3 shows the variation of solitary potential amplitude $(\phi)$ with $\xi$ for different values of the beam to hot electron temperature ratio $\left(T_{\mathrm{b}} / T_{\mathrm{h}}\right)$ as shown on the curves for Mach number $M=1.1$. Other parameters are same as in Fig. 1. It is observed that soliton amplitude decreases with the increase of $T_{\mathrm{b}} / T_{\mathrm{h}}$ values. As electron beam temperature approaches hot electron temperature, the soliton solution does not exist. Soliton solution exists for $0<T_{\mathrm{b}} / T_{\mathrm{h}}<0.16$. On the other hand, the soliton width

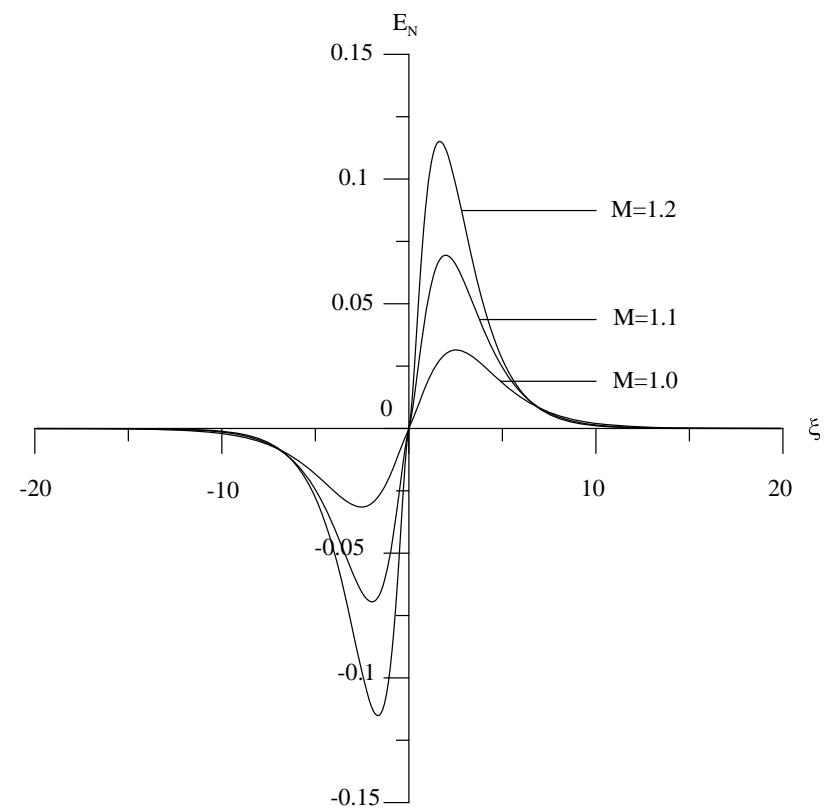

Fig. 2. Variation of normalized electric field $\left(E_{\mathrm{N}}\right)$ vs $\xi$ amplitude for the parameters of Fig. 1.

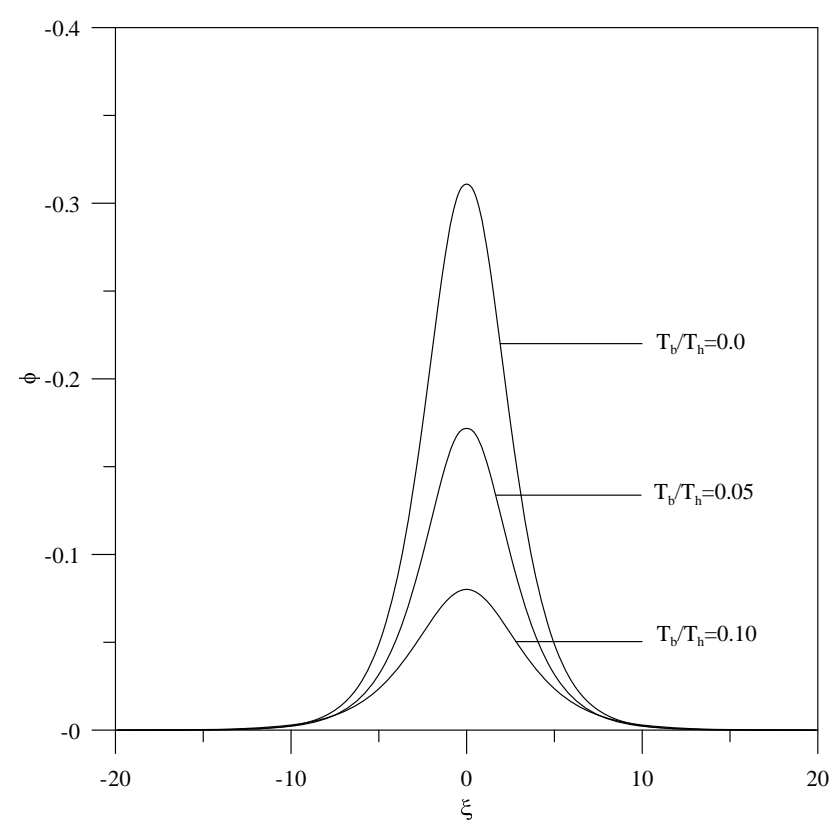

Fig. 3. Variation of soliton potential $\phi$ vs $\xi$ for electron beam to hot electron temperature ratio $\sigma_{\mathrm{b}}=T_{\mathrm{b}} / T_{\mathrm{h}}=0.0,0.05,0.1$ and Mach number $M=1.1$. Other parameters are same as in Fig. 1.

increases with the increase in $T_{\mathrm{b}} / T_{\mathrm{h}}$ values. Soliton amplitude is maximum in the presence of cold beam $\left(T_{\mathrm{b}} / T_{\mathrm{h}}=0\right)$. In Fig. 4 effect of electron beam speed is studied on the soliton amplitude. It is interesting to note that the soliton solution exists for both positive and negative values of $v_{\mathrm{o}} / v_{\mathrm{th}}$, 


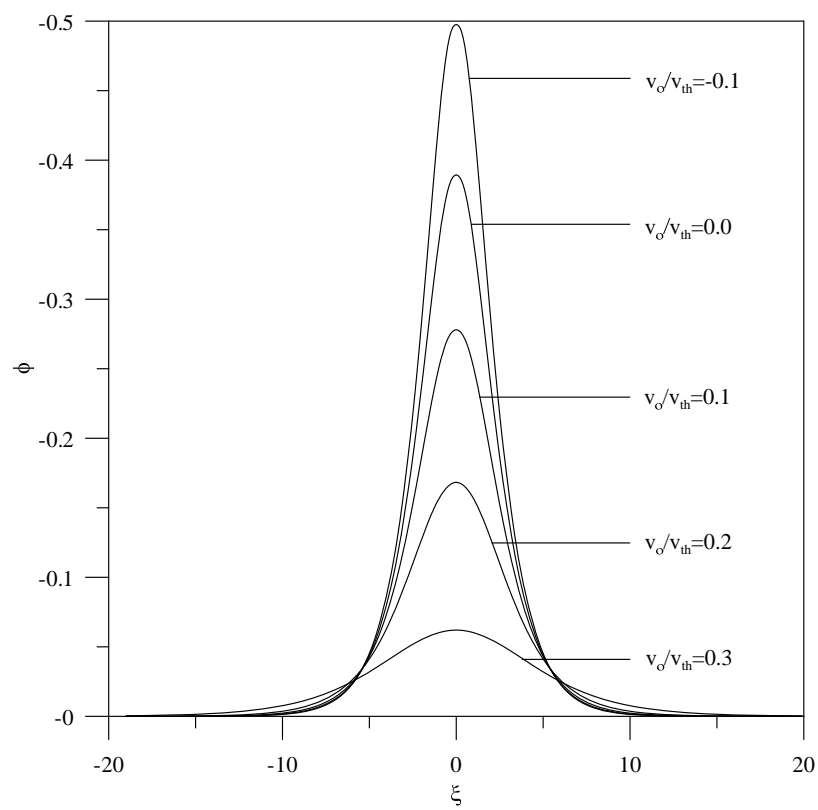

Fig. 4. Variation of soliton potential $\phi$ vs $\xi$ for normalized electron beam drift velocities $v_{\mathrm{o}} / v_{\text {th }}=-0.1,0.0,0.1,0.2$ and 0.3 for Mach number $M=1.1$. Other parameters are same as in Fig. 1 .

i.e. soliton solutions can be obtained for both the cases of co- and counter propagating electron beam to solitary wave propagation direction (cf. Fig. 4). Soliton solutions are found in the range $-0.14<v_{\mathrm{o}} / v_{\text {th }}<0.33$. Our theoretical model predicts the higher soliton amplitude in the case of counter propagating $\left(V+v_{\mathrm{o}}\right)$ electron beam than the co-propagating $\left(V-v_{\mathrm{o}}\right)$ electron beam. Our speculation is that this trend could be due to the more free energy available for the case of counter propagating electron beam than the co-propagating electron beam. However, width of the soliton is higher for co-propagating electron beam case and it increases with the increase in electron beam speed.

Figure 5 shows the effect of superthermal electrons on the solitary potential structures. Plots are shown for spectral index $\kappa=3,4$ and 8 for $M=1.1$ when the other parameters are taken the same as in Fig. 1. For low values of $\kappa$ (high superthermality), amplitude of the solitary structures is higher. Increasing the value of $\kappa$ leads to the decrease in soliton amplitude and attains a Maxwellian distribution at $\kappa \approx 30$. Width of solitons increases with the increase in $\kappa$ values.

Unnormalized values for the soliton velocity $(V)$, electric field $(E)$, soliton width $(W)$ and pulse duration $(\tau=W / V)$ are given in Tables 1, 2 and 3 for various values of spectral index, $\kappa, \sigma_{\mathrm{b}}=T_{\mathrm{b}} / T_{\mathrm{h}}$, and $v_{\mathrm{o}} / v_{\text {th }}$, respectively. The parameters are taken from an observed event in the dayside auroral region (Dubouloz et al., 1991, 1993). For this purpose first we have calculated the range of Mach numbers for which soliton solution exists. The lower limit of the Mach number is calculated from the critical Mach number $M_{\mathrm{o}}$ by solving

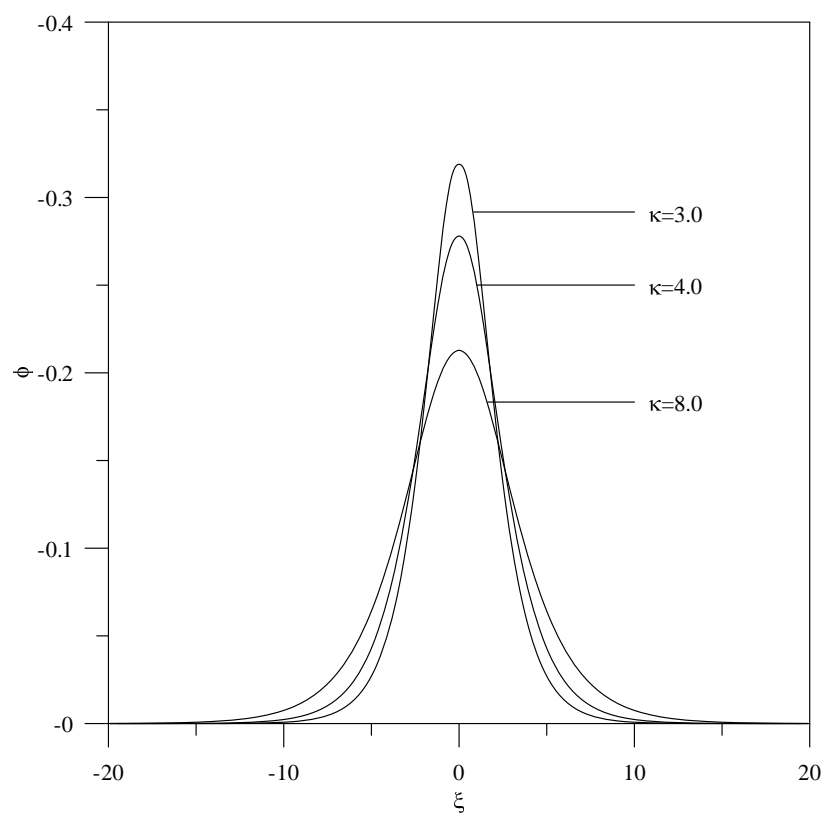

Fig. 5. Variation of soliton potential $\phi$ vs $\xi$ for kappa, $\kappa=3.0,4.0$ and 8.0 for $M=1.1$. Other parameters are same as in Fig. 1.

Table 1. Properties of electron-acoustic solitons such as soliton velocity $(V)$, electric field $(E)$, soliton width $(W)$ and pulse duration $(\tau)$ for various values of spectral index, $\kappa$ for the dayside auroral region parameters (Dubouloz et al., 1991, 1993); $N_{\mathrm{oc}}=0.2 \mathrm{~cm}^{-3}$, $N_{\mathrm{oh}}=1.5 \mathrm{~cm}^{-3}, N_{\mathrm{ob}}=1.0 \mathrm{~cm}^{-3}, \sigma_{\mathrm{c}}=T_{\mathrm{c}} / T_{\mathrm{h}}=T_{\mathrm{i}} / T_{\mathrm{h}}=\sigma_{\mathrm{i}}=$ $0.001, \sigma_{\mathrm{b}}=T_{\mathrm{b}} / T_{\mathrm{h}}=0.01, v_{\mathrm{o}} / v_{\text {th }}=0.1$, hot electron temperature, $T_{\mathrm{h}}=250 \mathrm{eV}$, cold electron temperature, $T_{\mathrm{c}}=2.5 \mathrm{eV}=T_{\mathrm{i}}$ (ion temperature).

\begin{tabular}{ccccc}
\hline$\kappa$ & $V\left(\mathrm{~km} \mathrm{~s}^{-1}\right)$ & $E\left(\mathrm{mV} \mathrm{m}^{-1}\right)$ & $W(\mathrm{~m})$ & $\tau=W / V(\mu \mathrm{s})$ \\
\hline 3.0 & $5370-7823$ & $2.6-479$ & $1288-256$ & $239-32.7$ \\
4.0 & $5834-8486$ & $6.1-544$ & $1159-280$ & $198-32.9$ \\
5.0 & $6099-8884$ & $9.9-595$ & $944-293$ & $154-32.9$ \\
\hline
\end{tabular}

the Eq. (15) for the relevant parameters and the higher limit is found numerically by solving the Sagdeev pseudo potential $V\left(\phi_{m}, M\right)=0$ in Eq. (14) for various values of $M>M_{\mathrm{o}}$ (as shown in Fig. 1, for example). The highest value of $M$ beyond which soliton solutions are not found is $M_{\max }$ and the corresponding amplitude of the soliton is $\phi_{\mathrm{m}}$. Unnormalizing the Mach number gives us the soliton velocity. The energy Eq. (13) is solved numerically by using the Euler's method to calculate the soliton electric field as shown in Tables 1-3. Subsequently, width is calculated by using the method of full width at half maximum.

Table 1 clearly shows that soliton velocity, electric field amplitude tend to increase with $\kappa$ but width and pulse duration seems to decrease. Also, the range of soliton velocities tends to increase with $\kappa$. This result is similar to Devanandhan et al. (2011). It is noted from Table 2 that the 
Table 2. Properties of electron-acoustic solitons such as soliton velocity $(V)$, electric field $(E)$, soliton width $(W)$ and pulse duration ( $\tau$ ) for various values of $\sigma_{\mathrm{b}}=T_{\mathrm{b}} / T_{\mathrm{h}}$, for the dayside auroral region parameters (Dubouloz et al., 1991, 1993); $N_{\mathrm{oc}}=0.2 \mathrm{~cm}^{-3}$, $N_{\mathrm{oh}}=1.5 \mathrm{~cm}^{-3}, N_{\mathrm{ob}}=1.0 \mathrm{~cm}^{-3}, \sigma_{\mathrm{c}}=T_{\mathrm{c}} / T_{\mathrm{h}}=T_{\mathrm{i}} / T_{\mathrm{h}}=\sigma_{\mathrm{i}}=$ $0.001, \kappa=4, v_{\mathrm{o}} / v_{\mathrm{th}}=0.1$, hot electron temperature, $T_{\mathrm{h}}=250 \mathrm{eV}$, cold electron temperature, $T_{\mathrm{c}}=2.5 \mathrm{eV}=T_{\mathrm{i}}$ (ion temperature).

\begin{tabular}{lcccc}
\hline$\sigma_{\mathrm{b}}=T_{\mathrm{b}} / T_{\mathrm{h}}$ & $V\left(\mathrm{~km} \mathrm{~s}^{-1}\right)$ & $E\left(\mathrm{mV} \mathrm{m}^{-1}\right)$ & $W(\mathrm{~m})$ & $\tau=W / V(\mu \mathrm{s})$ \\
\hline 0.00 & $5635-17900$ & $1.5-3788$ & $1831-246$ & $324-13.7$ \\
0.01 & $5834-8486$ & $6.1-544$ & $1159-280$ & $198-32.9$ \\
0.05 & $6298-7558$ & $6.5-193$ & $944-324$ & $149-42.8$ \\
0.10 & $6895-7691$ & $10.6-111$ & $744-359$ & $107-46.6$ \\
0.15 & $7425-7955$ & $12.4-72$ & $600-369$ & $80.8-46.3$ \\
\hline
\end{tabular}

maximum electric field amplitude of the soliton for the case of $\sigma_{\mathrm{b}}=T_{\mathrm{b}} / T_{\mathrm{h}}=0$ (cold electron beam) is about $4 \mathrm{~V} \mathrm{~m}^{-1}$ which is on the higher side for auroral region of the Earth's magnetosphere. However, for finite values of $T_{\mathrm{b}} / T_{\mathrm{h}}$, e.g. say $T_{\mathrm{b}} / T_{\mathrm{h}}=0.01$, the maximum electric field amplitude is about $544 \mathrm{mV} \mathrm{m}^{-1}$, and it subsequently decreases with further increase in $T_{\mathrm{b}} / T_{\mathrm{h}}$ value. Also, the range of soliton velocities and pulse duration decreases with increased beam to hot electron temperature. From Table 3, it can be seen that the range of soliton velocities and pulse duration decrease with the increase in $v_{\mathrm{o}} / v_{\text {th }}$ values. Maximum amplitude occurs for counter propagating electron beam and it decreases subsequently for the co-propagating electron beam.

\section{Discussion}

We have studied electron-acoustic solitons in four component plasma consisting of cold electrons and ions, hot superthermal electrons and an electron beam. It is found that the inclusion of electron beam significantly modifies the regime for the existence of solitons.

For the auroral region parameters (Dubouloz et al., 1991, 1993), namely, hot electron temperature, $T_{\mathrm{h}}=250 \mathrm{eV}, N_{\mathrm{oc}}=$ $0.2 \mathrm{~cm}^{-3}, N_{\mathrm{oh}}=1.5 \mathrm{~cm}^{-3}, N_{\mathrm{ob}}=1.0 \mathrm{~cm}^{-3}, T_{\mathrm{c}} / T_{\mathrm{h}}=0.001$, $T_{\mathrm{i}} / T_{\mathrm{h}}=0.001, T_{\mathrm{b}} / T_{\mathrm{h}}=0.01, M=1.1$ and $\kappa=4$, the electric field amplitude of solitons comes out to be (524-30) $\mathrm{mV} \mathrm{m}^{-1}$ for electron beam speed (660-1990) $\mathrm{km} \mathrm{s}^{-1}$ (this corresponds to $\left.v_{\mathrm{o}} / v_{\text {th }}=(-0.1-0.3)\right)$. The corresponding width is found to be in the range (329-729) $\mathrm{m}$.

Inclusion of an electron beam affects the regime of the soliton existence. For example, Mach number for which soliton solutions are obtained for various $\kappa$ (cf. Table 1) is pushed to the higher side in the present four component plasma model with electron beam as compared to threecomponent model (cf. Table 1 of Devanandhan et al., 2011). Similarly the effect of electron beam temperature is to lower the electric field amplitude, soliton velocities and pulse duration significantly as is evident from Table 2. Further, effect of electron beam speed is similar to electron beam temperature
Table 3. Properties of electron-acoustic solitons such as soliton velocity $(V)$, electric field $(E)$, soliton width $(W)$ and pulse duration $(\tau)$ for various values of $v_{\mathrm{o}} / v_{\mathrm{th}}$, for the dayside auroral region parameters (Dubouloz et al., 1991, 1993); $N_{\mathrm{oc}}=0.2 \mathrm{~cm}^{-3}$, $N_{\mathrm{oh}}=1.5 \mathrm{~cm}^{-3}, \quad N_{\mathrm{ob}}=1.0 \mathrm{~cm}^{-3}, \quad \sigma_{\mathrm{c}}=T_{\mathrm{c}} / T_{\mathrm{h}}=T_{\mathrm{i}} / T_{\mathrm{h}}=\sigma_{i}=0.001$, $\kappa=4, \sigma_{\mathrm{b}}=T_{\mathrm{b}} / T_{\mathrm{h}}=0.01$, hot electron temperature, $T_{\mathrm{h}}=250 \mathrm{eV}$, cold electron temperature, $T_{\mathrm{c}}=2.5 \mathrm{eV}=T_{\mathrm{i}}$ (ion temperature).

\begin{tabular}{lcccc}
\hline$v_{\mathrm{o}} / v_{\text {th }}$ & $V\left(\mathrm{~km} \mathrm{~s}^{-1}\right)$ & $E\left(\mathrm{mV} \mathrm{m}^{-1}\right)$ & $W(\mathrm{~m})$ & $\tau=W / V(\mu \mathrm{s})$ \\
\hline-0.1 & $4641-7956$ & $1.7-715$ & $1559-263$ & $336-33.1$ \\
0.0 & $5238-8088$ & $4.7-595$ & $1101-283$ & $210-34.9$ \\
0.1 & $5834-8486$ & $6.1-544$ & $1159-280$ & $198-32.9$ \\
0.3 & $7094-9613$ & $8.5-518$ & $1001-277$ & $141-28.8$ \\
\hline
\end{tabular}

as can be seen in Table 3. The range of kappa values also widens due to presence of electron beam and minimum value of kappa for which soliton solutions are obtained are higher as compared to three-component model of Devanandhan et al. (2011).

For the parameters discussed in this paper, we did not find positive polarity solitons. This could be due to the restrictions on the plasma parameters since we are using the fixed densities of the cold, hot and beam electrons as observed by the Viking satellite in the auroral region. However, the possibility of positive polarity solitons can not be ruled out if one carry out the analysis by relaxing kappadistribution for the hot electrons and allows the hot electron inertia to be retained in the analysis as suggested by Verheest et al. $(2005,2007)$ and subsequently shown by Lakhina et al. $(2009,2011)$. In multi-component plasmas, for positive polarity electron-acoustic solitons to occur the cold electron density should to be of the order of or greater than hot electron density as has been seen in the literature (Lakhina et al., 2008a). In our case, the cold electron density is smaller than the hot electron density.

Our results may be useful in understanding the electrostatic solitary waves in the auroral region of the Earth's magnetosphere where such electrostatic solitary structures with similar electric field amplitudes have been observed (Ergun et al., 1999).

Acknowledgements. GSL thanks the Indian National Science Academy, New Delhi, India, for the support under the Senior Scientist scheme. GSL and SVS thank University of the Western Cape, Bellville, South Africa for the hospitality during their visit.

Edited by: P.-L. Sulem

Reviewed by: F. Verheest and another anonymous referee 


\section{References}

Armstrong, T. P., Paonessa, M. T., Bell II, E. V., and Krimigis, S.M.: Voyager observations of Saturnian ion and electron phase space densities, J. Geophys. Res., 88, 8893-8904, 1983.

Berthomier, M., Pottelette, R., Malingre, M., and Khotyaintsev, Y.: Electron-acoustic solitons in an electron-beam plasma system, Phys. Plasmas, 7, 2987-2994, 2000.

Berthomier, M., Pottelette, R., Muschietti, L., Roth, I., and Carlson, C. W.: Scaling of 3D solitary waves observed by FAST and POLAR, Geophys. Res. Lett., 30, 2148, doi:10.1029/2003GL018491, 2003.

Cattell, C., Bergmann, R., Sigsbee, K., Carlson, C., Chatson, C., Ergun, R., McFadden, J., Mozer, F. S., Temerin, M., Strangeway, R., Elphic, R., Kistler, L., Moebius, E., Tang, L., Klumpar, D., and Pfaff, R.: The association of electrostatic ion cyclotron waves, ion and electron beams and field-aligned currents: FAST observations of an auroral zone crossing near midnight, Geophys. Res. Lett., 25, 2053-2056, 1998.

Devanandhan, S., Singh, S. V., and Lakhina, G. S.: Electron acoustic solitons with kappa-distributed electrons, Phys. Scr., 84, 025507, doi:10.1088/0031-8949/84/02/025507, 2011.

Dubouloz, N., Pottelette, R., Malingre, M., Holmgren G., and Lindqvist, P. A.: Detailed analysis of broadband electrostatic noise in the dayside auroral zone, J. Geophys. Res., 96, 35653579, 1991.

Dubouloz, N., Treumann, R. A., Pottelette R., and Malingre, M.: Turbulence generated by a gas of electron acoustic solitons, J. Geophys. Res., 98, 17415-17422, 1993.

El-Shewy, E. K.: Higher-order solution of an electron-acoustic solitary waves with non-thermal electrons, Chaos, Solitons Fractals, 34, 628-638, doi:10.1016/j.chaos.2006.03.103, 2007.

Ergun, R. E., Carlson, C. W., Muschietti, L., Roth, I., and McFadden, J. P.: Properties of fast solitary structures, Nonlin. Processes Geophys., 6, 187-194, doi:10.5194/npg-6-187-1999, 1999.

Gill, T. S., Kaur, H., and Saini, N. S.: Small amplitude electron-acoustic solitary waves in a plasma with nonthermal electrons. Chaos, Solitons Fractals, 30, 1020-1024, doi:10.1016/j.chaos.2005.09.070, 2006.

Hellberg, M. A. and Mace, R. L.: Generalized plasma dispersion function for a plasma with a kappa-Maxwellian velocity distribution, Phys. Plasmas, 9, 1495-1504, doi:10.1063/1.1462636, 2002.

Kakad, A. P., Singh, S. V., Reddy, R. V., Lakhina, G. S., Tagare, S. G., and Verheest, F.: Generation mechanism for electron acoustic solitary waves, Phys. Plasmas, 14, 052305, doi:10.1063/1.2732176, 2007.

Kakad, A. P., Singh, S. V., Reddy, R. V., Lakhina, G. S., and Tagare, S. G.: Electron acoustic solitary waves in the Earth's magnetotail region, Adv. Space. Res., 43, 1945-1949, doi:10.1016/j.asr.2009.03.005, 2009.

Lakhina, G. S., Kakad, A. P., Singh, S. V., and Verheest, F.: Ionand electron-acoustic solitons in two-electron temperature space plasmas, Phys. Plasmas, 15, 062903, doi:10.1063/1.2930469, 2008a.

Lakhina, G. S., Singh, S. V., Kakad, A. P., Verheest, F., and Bharuthram, R.: Study of nonlinear ion- and electron-acoustic waves in multi-component space plasmas, Nonlin. Processes Geophys., 15, 903-913, doi:10.5194/npg-15-903-2008, 2008 b.

Lakhina, G. S., Singh, S. V., Kakad, A. P., Goldstein, M. L., Vinas,
A. F., and Pickett, J. S.: A mechanism for electrostatic solitary structures in the Earth's magnetosheath, J. Geophs. Res., 114, A09212, doi:10.1029/2009JA014306, 2009.

Lakhina, G. S., Singh, S. V., and Kakad, A. P.: Ion- and electronacoustic solitons and double layers in multi-component space plasmas, Adv. Space Res., 47, 1558-1567, 2011.

Leubner, M. P.: On Jupiter's Whistler emission, J. Geophys. Res., 87, 6335-6338, 1982.

Mace, R. L. and Hellberg, M. A., A dispersion function for plasmas containing superthermal particles, Phys. Plasmas, 2, 2098-2109, 1995.

Mace, R. L. and Hellberg, M. A.: The Korteweg-de VriesZakharov-Kuznetsov equation for electron-acoustic waves, Phys. Plasmas, 8, 2649-2656, doi:10.1063/1.1363665, 2001.

Mace, R. L., Baboolal, S., Bharuthram, R., and Hellberg, M. A.: Arbitrary-amplitude electron-acoustic solitons in a two-electroncomponent plasma, J. Plasma Phys., 45, 323-338, 1991.

Mace, R. L., Amery, G., and Hellberg, M. A.: The electron-acoustic mode in a plasma with hot suprathermal and cool Maxwellian electrons, Phys. Plasmas, 6, 44-49, 1999.

Marsch, E., Muhlhauser, K. H., Schwenn, R., Rosenbauer, H., Pillip W., and Neubauer, F. M.: Solar wind protons: Three-dimensional velocity distributions and derived plasma parameters measured between 0.3 and 1 AU, J. Geophys. Res., 87, 52-72, 1982.

Miyake, T., Omura, Y., and Matsumoto, H.: Electrostatic particle simulations of solitary waves in the auroral region, J. Geophys. Res., 105, 23239-23249, 2000.

Pickett, J. S., Chen, L.-J., Kahler, S. W., Santolík, O., Goldstein, M. L., Lavraud, B., Décréau, P. M. E., Kessel, R., Lucek, E., Lakhina, G. S., Tsurutani, B. T., Gurnett, D. A., CornilleauWehrlin, N., Fazakerley, A., Rème, H., and Balogh, A.: On the generation of solitary waves observed by Cluster in the nearEarth magnetosheath, Nonlin. Processes Geophys., 12, 181-193, doi:10.5194/npg-12-181-2005, 2005.

Pottelette, R., Ergun, R. E., Truemann, R. A., Berthomier, M, Carlson, C. W., McFadden, J. P., and Roth, I.: Modulated electronacoustic waves in auroral density cavities: FAST observations, Geophys. Res. Lett., 26, 2629-2632, 1999.

Sahu, B.: Electron acoustic solitary waves and double layers with superthermal hot electrons, Phys. Plasmas, 17, 122305, doi:10.1063/1.3527988, 2010.

Singh, S. V. and Lakhina, G. S.: Electron acoustic solitary waves with non-thermal distribution of electrons, Nonlin. Processes Geophys., 11, 275-279, doi:10.5194/npg-11-275-2004, 2004.

Singh, S. V., Reddy, R. V., and Lakhina, G. S.: Broadband electrostatic noise due to nonlinear electron-acoustic waves, Adv. Space. Res., 28, 1643-1648, 2001.

Summers, D. and Thorne, R. M.: The modified plasma dispersion function, Phys. Fluids B, 3, 1835-1847, 1991.

Thorne, R. M. and Horne, R. B.: Landau damping of magnetospherically reflected whistlers, J. Geophys. Res., 99, 17249-17258, 1994.

Thorne, R. M. and Summers, D.: Landau damping in space plasmas, Phys. Fluids B, 3, 2117-2123, 1991a.

Thorne, R. M. and Summers, D.: Enhancement of wave growth for warm plasmas with a high-energy tail distribution, J. Geophys. Res., 96, 217-223, 1991b.

Vasyliunas, V. M.: A survey of low-energy electrons in the evening sector of the magnetosphere with OGO 1 and OGO 3, J. Geo- 
phys. Res., 73, 2839-2884, 1968.

Verheest, F.: Obliquely propagating large amplitude solitary waves in charge neutral plasmas, Nonlin. Processes Geophys., 14, 4957, doi:10.5194/npg-14-49-2007, 2007.

Verheest, F., Cattart, T., and Hellberg, M. A.: Compressive and rarefactive electron-acoustic solitons and double layers in space plasmas, Space Sci. Rev., 121, 299-311, doi:10.1007/s11214006-4148-7, 2005.
Younsi, S. and Tribeche, M.: Arbitrary amplitude electron-acoustic solitary waves in the presence of excess superthermal electrons, Astrophys. Space Sci., 330, 295-300, doi:10.1007/s10509-0100404-0, 2010. 